\title{
Emerging technology for bioanalysis in the next decade
}

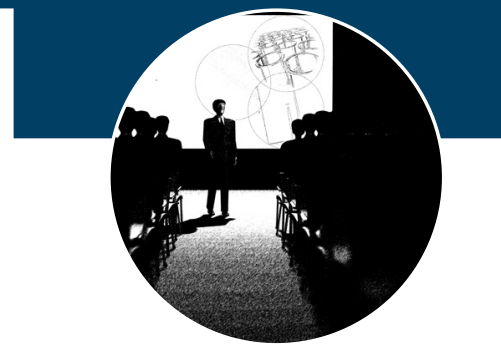

The 13th Annual University of Wisconsin Land O'Lakes Bioanalytical Conference 16-20 July 2012, Merrimac, WI, USA

This University of Wisconsin School of Pharmacy bioanalytical conference is presented each year by the Extension Services in Pharmacy, the professional development department within the School. The purpose of this 4-day conference is to provide an educational forum to discuss issues and applications associated with the analysis of xenobiotics, metabolites, biologics and biomarkers in biological matrices. The conference is designed to include and encourage an open exchange of scientific and methodological applications for bioanalysis. To increase the interactive nature of the conference, the program was a mixture of lectures, poster sessions, round table discussions and workshops. This article summarizes the presentations at the 13th Annual Conference.

The 13th Annual Land O'Lakes Bioanalytical Conference, titled 'Emerging technology for bioanalysis in the next decade', was held on 16-20 July 2012 at Devil's Head Resort in Merrimac, WI, USA. This conference brings together an international group of bioanalytical scientists with the express intent of discussing cutting-edge science for small- and largemolecule bioanalysis in a relaxed atmosphere that also promotes networking opportunities. The conference provides an educational forum with presentations by scientific leaders from academia, industry, contract laboratories and regulatory authorities.

The success of the program is due to the work of the Planning Committee, which consists of scientists involved with bioanalysis on a daily basis, and their ability to identify timely topics and presenters who are experts in the field. Specific objectives for the conference and a list of the Planning Committee members can be found at the conference website [1].

\section{Four plenary sessions}

Morning meetings consisted of four plenary sessions focusing on specific themes associated with bioanalytical issues. The first plenary session was titled 'Imaging, organ targeting and quantitation of drugs'. Stacy Ho (Genzyme Corporation, MA, USA) gave an overview on quantitation of drugs (including their metabolites and biomarkers) in tissues. She discussed the analysis workflow, highlighted the similarities and differences between tissue and plasma analysis, and listed good practices for effective and efficient method development and successful method qualification. She provided specific examples to illustrate that the impact of using perfusion to remove blood from tissue during the tissue collection should be evaluated because it is compound- (especially biomarker) and tissuedependent. Ho demonstrated that it is important to evaluate tissue homogenization, method accuracy, precision and calibration range, as well as analyte interference and stability for each tissue matrix independently when quantifying the same analyte in multiple tissues. Ho also discussed methodologies for determination of free drug concentrations in tissues.

Christine Huang (Bristol-Myers Squibb, NJ, USA) articulated the reasons to, or not to, monitor tissue exposures, showed case studies that actually implemented these strategies, and explained the lessons learned to determine the best pathway forward. One case study exemplified the utility of PK and PD data, such as receptor occupancy, in vitro potency, in vivo $\mathrm{IC}_{50}$ values and free plasma concentrations, to revise compound screening paradigms to accelerate compound evaluation. Other case studies were presented that demonstrated the most ideal time to change a strategic approach and limit tissue sample collection and protein-binding measurement; confirmed compound target access by using tissue distribution data via PET imaging and brain uptake studies; verified the importance of liver and muscle exposure data for efficacy determination, as well as toxicity evaluation, during the selection of the most potent and safe compounds; and exemplified the utility of PK and quantitative whole-body autoradiography studies to elucidate the marginal role volume of distribution played when comparing diet-induced obesity rats versus lean
James E DeMuth*1, Qin C $\mathrm{Ji}^{2}$, Brian P Booth ${ }^{3}$, Eric N Fluhler $^{4}$, Roger Greathead ${ }^{5}$, Stacy $\mathrm{Ho}^{6}$, R John Stubbs ${ }^{7}$ \& Douglas J Turk ${ }^{8}$

'University of Wisconsin, 777 Highland Ave, Madison, WI 53705, USA ${ }^{2}$ Bristol-Myers Squibb Company, NJ, USA

${ }^{3}$ Center for Drug Research \& Evaluation, US FDA, MD, USA ${ }^{4}$ Pfizer Research, NY, USA

${ }^{5}$ PRA International, KS, USA ${ }^{6}$ Genzyme Corporation, MA, USA ${ }^{7}$ Stubbs \& Hensel Pharma Consulting, LLC, PA, USA

${ }^{8}$ PRACS Institute, Toronto, Ontario, Canada

*Author for correspondence:

E-mail: jedemuth@pharmacy.wisc.edu 
rats. Huang illustrated why tissue exposure data had decisive values at different stages of drug development, and why PK/PD modeling is a valuable scientific tool to decide when to collect tissue samples. The third presentation by C Andrew Boswell (Genentech, CA, USA) highlighted the importance of studying tissue uptake of antibodies at a compartmental level. Monoclonal antibodies have provided many validated and potential new therapeutic candidates for various diseases in neurology, ophthalmology, immunology and oncology. Many antigens reside within the interstitial space. Boswell gave a brief overview of receptor occupancy and its interpretation in radiotracer studies. Concepts relevant to the kinetics and behavior of antibodies within the interstitium were discussed, with a special emphasis on radiometric measurement of quantitative pharmacology. Molecular probes were reviewed, including chemical techniques, selection criteria, data interpretation and relevance to the study of antibody PK. Experimental methods for measuring the spatial composition of tissues were examined in terms of relative vascular, interstitial and cellular volumes using solids. Boswell outlined the available methods for PK measurements of antibodies and physiological measurements of the occupied compartments, while emphasizing such approaches may not fully capture the complexities of dynamic, heterogeneous tumors and other tissues.

The last presentation by M Reid Groseclose (GlaxoSmithKline, NC, USA) gave an overview of matrix-assisted laser desorption/ionization (MALDI) imaging MS (IMS) and its applications in drug development. He focused on the sample preparation steps involved in conducting a MALDI-IMS experiment and highlighted both the advantages and challenges of this technology. Groseclose showed examples where MALDI-IMS was used to provide valuable drug and metabolite distribution data. Examples in the presentation included the distribution analysis of a drug and its metabolites in liver tissue and the correlation with observed hepatotoxicity and the comparative CNS penetration and metabolism of a compound and its metabolites in different preclinical models. The final part of the presentation presented the latest progress in developing a quantitation model that can be used to directly quantitate drugs and metabolites in a MALDI-IMS experiment.

The focus of the second plenary session was on ultra low-volume sampling in bioanalysis. The first speaker, Elizabeth Mahan (Merck, PA, USA), provided an overview of analytical issues associated with liquid microsampling. She noted that some of the driving forces for the removal of small quantities of samples $(<100 \mu \mathrm{l})$ for analysis include animal welfare ethical considerations, regulatory requirements in children, as well as increased analytical capacities and serial blood collection requirements for longer periods associated with biologics. A number of examples were presented illustrating the advantages of microsampling techniques with a detailed comparison of blood microsampling for DBS sample analysis. Mahan described Merck's recent efforts for automation of microsampling techniques, including operation of a liquid microjunction surface sampling probe (LMJSSP) using Advion's liquid extraction surface analysis system, as well as blood microsampling using the Instech $\mathrm{ABS} 2^{\mathrm{TM}}$ automated blood sampling instrument. The second presentation of this plenary session focused on miniaturization of sample handling in bioanalysis using solid-phase microextraction (SPME). Janus Pawliszyn (University of Waterloo, Ontario, Canada) reviewed his extensive research with SPME techniques. He described the features of various SPME configurations, including new metal fiber SPME assemblies and commercially available SPME devices. Automation strategies, including a 96-blade SPME system, a 96-well thin film SPME system, as well as a summary of the available biocompatible SPME coatings were also presented. Pawliszyn also reviewed a number of analytical approaches where SPME microsampling techniques might be utilized, including in vivo SPME, kinetic or equilibria studies, metabolomics and future clinical studies. The next presentation by Thorsten Verch (Merck, PA, USA) focused on large-molecule sample analysis using immunoassay with Gyros. Verch described the Gyros system as a CD-based immunoassay platform based on miniature streptavidin columns. After reviewing the basic operating principles and applications for the Gyros unit, he described the utilization of the system for drug capture-mediated PK assays, generic human monoclonal antibody PK assays and as a potential reagent screening tool for vaccine assays. It should be noted that the Gyrolab unit usually compared favorably with conventional plate-based ELISA methods while utilizing less sample, with its main drawback a loss in overall sensitivity. The final presentation of the second plenary session was an update and progress report on dry matrix spots (DBS) from the perspective of Aziz Fillali-Ansary (Sanofi, France). Following a brief historical review of 
Sanofi's experience with DBS, he presented extensive data on the correlation between DBS results and plasma results for a number of clinical studies. He noted that the additional tests required for DBS method validations included stability, hemolysis testing and puncher carryover, as well as blood volume and punch position tests. Fillali-Ansary suggested that the use of a fixed volume and whole spot method for sample collection would eliminate the concern of any hematocrit errors associated with standard partial punches. He indicated that all of Sanofi's Phase I studies are currently being supported with DBS analysis.

The Thursday morning plenary session was titled 'Third plenary session: how low is low enough for quantitation?' Andrew Vick (Seventh Wave Laboratories, MO, USA) discussed the evolution of PK and the rapid advancements in bioanalysis leading to sensitivities in the $\mathrm{pg} / \mathrm{ml}$ with the introduction of UPLC-MS/MS technology. He reviewed some of the fundamental PK calculations and the differing needs from discovery (medium sensitivity) through to full development (higher sensitivity). Using several examples, he explained that very high sensitivity assays were not always needed and might provide misleading PK data. For many metabolite assays, and even for pivotal bioavailability/bioequivalence studies, ng/ $\mathrm{ml}$ sensitivities are often adequate. The conclusion was that developing assays to meet the needs of the study being supported was critical rather than trying to achieve very LLOQs. Richard King (PharmaCadence Analytical Services, LLC, PA, USA) discussed the investigation of lower LC rates and smaller internal diameter columns in quantitative LC-MS/MS assays using ESI. He reviewed the fundamentals of what was actually happening in the instrument and made comparisons with UV detection, which is a concentrationdependent detector. He differentiated peak area measurements as the total mass of the analyte detected compared with peak area, which represents the maximum mass flow rate of the analyte reaching the detector. King discussed the fundamentals of HPLC separation and compared the internal diameter of the columns. Flow rate has a more profound effect in response for peptides than for small molecules. Rand Jenkins (PPD, VA, USA) discussed endogenous measurements and ways to achieve LLOQs. Typical values were quoted as $0.1-1 \mathrm{pg} / \mathrm{ml}$ for a $500 \mathrm{Da}$ compound and $1-10 \mathrm{pg} / \mathrm{ml}$ for a $5 \mathrm{kDa}$ compound, mainly due to recent advances in LC-MS/MS technology. Sample extraction was cited as a common problem for all analytes in achieving very
LLOQs leading to background interference, carryover and ion suppression. Jenkins emphasized the need for cleanliness in all phases of very high-sensitivity assays. He discussed the benefits of the surrogate analyte approach by fortifying natural matrix with 'heavy' stable isotope-labeled analyte with several examples. Jenkins also discussed the approach to measuring biomarkers for Alzheimer's disease. Jeffrey Sailstad (Sailstad and Associates, NC, USA) discussed sensitivity in immunogenicity assessments. Greater diversity in the design and delivery for biotherapeutics makes the analytical measurements more challenging, but assays continue to improve. He discussed false positives and the US FDA need for statistical analysis above 5\%. Assigning appropriate cut points is required in all assays. In several examples he stressed the need to remove the 'free endogenous component' in these assays to improve sensitivity by approximately ten-times.

The conference concluded on Friday with a session on US FDA Inspections. Sam Haidar, the branch chief for the Bioequivalence group in the FDA Office of Scientific Investigations presented the approach used by Office of Scientific Investigations for bioequivalence inspections and briefly described the new surveillance model that is being developed. Specifically, Haidar described the reasons for conducting routine and 'for cause' inspections, namely to ensure compliance with appropriate regulations, ensure data quality and integrity, and to ensure adequate documentation to allow reconstruction of the study. Haidar concluded by providing several anonymous examples of inspection failures, which included multiple reanalysis of a given set of samples and the use of study samples to equilibrate the system prior to their analysis. The take-home message was that the intended use of the patient samples dictate which regulations will apply. Jeff Moran (QPS, DE, USA) closed the session with a lighthearted but realistic examination of myths commonly associated with FDA inspections. The message was that the scientist needs to understand what the regulations are and where they specifically apply. For instance, he pointed out that the GLP requirements of 21 CFR 58 apply to nonclinical studies, but not to studies in humans. Moran pointed out that 21 CFR 320 applies to human studies and necessitates method validation to assure data quality. He concluded his presentation with the message that scientists should use good judgment in validating methods for studies in humans according to the fit-for-purpose concept. 


\section{Evening sessions}

During the Tuesday evening session, Joanna Zheng (Bristol-Myers Squibb, NJ, USA) discussed the challenges and applications of using LC-MS/MS assay for the quantitative determination of therapeutic proteins in drug discovery. Using examples and working through all the stages of her process of in silico protein digestion to identify target peptide fragments to measure, internal standard strategies, sample preparation, LC-MS/MS method development and matrix effects, she was able to convey the challenges and successful approaches she has applied. Zheng demonstrated that high specificity, linearity and fast assay development could be achieved using LC-MS/MS, which is particularly useful in cases where ligand binding is not applicable or cannot be applied.

The analytical investigators forum was held on Thursday evening and provided a platform for scientists to present research building on the major themes of the conference. Long Yuan (Bristol-Myers Squibb, NJ, USA) presented the feasibility and applicability of automation for standard and quality control sample preparation in DBS methods. Using buspirone as a test compound, he presented data that showed similar precision and accuracy could be achieved using manual pipetting and a Tecan automated liquid handler for blood spotting. Critical factors to acceptable precision were identified as spot volume, tip height and dispensing volume. His work also demonstrated the successful preparation of whole blood standards and quality controls by two automated approaches, direct spiking and serial dilution. Yuhong Xiang (Biogen Idec, MA, USA) presented measurements for biologics using DBS. She presented development and characterization of an antidrug antibody assay for a murine chimeric therapeutic. The study included the use of design of experiments to optimize assay conditions such as paper type, elution buffer, spot volume and punch size. Subsequent method qualification results demonstrated extraction efficiency of over 90\% (compared with whole blood), inter- and intra-card precision of $<15 \%$ and good correlation between plasma and DBS ELISA results, with similar drug tolerance and sensitivity when compared with the plasma method. Todd Reimer (Abbott, IL, USA) presented validation and application of a method for the determination of zotarolimus in various tissues following stent application. Standard and QC spiking strategies and the development of a generalized extraction method (based on a pre-existing whole blood assay) were reviewed. Validation and in-study performance results suggested sub-nanogram LOQ's could be obtained for numerous tissues with adequate precision and accuracy.

\section{Workshops \& round table discussions}

Tuesday afternoon's session complemented the morning plenary session and featured case studies on tissue method development. Hong Gao (Vertex, MA, USA) used case studies to demonstrate that perfusion of tissues can be critical to the success of analysis for certain organs, and the impact of perfusion should be evaluated during method development for critical studies. He summarized commonly used tissue treatment techniques and provided guidelines for technique selection based on the size and texture of the tissues. He further discussed that foams in the tissue homogenates can impact sample homogeneity and data quality. Case studies indicated that the addition of antifoaming agent could cause ion suppression for LC-MS/MS analysis and centrifugation of homogenates may lead to underestimating the concentrations. He presented typical approaches for selecting surrogate matrix and showed that internal standard response can be used to check the suitability of surrogate matrix. Finally, he discussed that fit-for-purpose is essential when developing methods for tissue sample analysis. Brad Yuska (QPS, DE, USA) presented a universal method where multiple types of tissue samples can be extracted efficiently and quantified against a single standard curve. The method used 2D LC to address the matrix effects during LC-MS/MS analysis while maintaining high throughput. The method was able to eliminate and normalize the matrix effects of multiple tissues for paclitaxel, docetaxel and azidothymidine. The use of radiolabeled paclitaxel and azidothymidine to evaluate recovery of the homogenization and extraction procedures is being conducted and the results will be compared with those of LC-LC-MS/MS. The results for paclitaxel had low recovery for all tissues and the potential formation of metabolite will be studied in the future.

The Thursday afternoon workshop considered problems commonly encountered in daily laboratory work. The workshop was facilitated by Qin Ji (Bristol-Myers Squibb, NJ, USA) and Jeffrey Sailstad (Sailstad and Associates, NC, USA) and covered a broad range of questions. Each question was discussed and best-practices were shared by attendees for discussion, 
refinement and education (see outcomes at the aforementioned website). Discussion was highly interactive, with many attendees gaining new understanding of experiments that could be added to their own laboratories.

Wednesday evening, following the poster session, informal round tables were held on several topics. These discussions and facilitators covered: GBC A7: repeat analysis and ISR (Eric Fluhler, Pfizer, CT, USA); GBC L4: critical reagents and stability for LBAs (Priya Sriraman, HoffmanLaRoche, NJ, USA); small-molecule analysis issues (Stacy Ho, Genzyme Corporation, MA, USA); and bioanalytical outsourcing (Randall Guthrie, RHG and Associates, WI, USA).

\section{Conclusion}

The informal, isolated setting, was designed to allow attendees more time to meet each other and discuss their organizations, practices and the science. Feedback was typically very positive and a summary of the evaluation results

\section{Websites}

1 13th Annual Land O'Lakes Bioanalytical Conference.

http://ce.pharmacy.wisc.edu/ courseinfo/2012JulyLOL are presented on the website for the 2013 program. Major changes are planned for next year's conference, which is currently being developed and will be posted on the University's website when finished [2].

\section{Disclaimer}

BP Booth would like to state that the views expressed are those of the authors and do not reflect official policy of the US FDA. No official endorsement by the FDA is intended or should be inferred.

Financial \& competing interests disclosure The authors have no relevant affiliations or financial involvement with any organization or entity with a financial interest in or financial conflict with the subject matter or materials discussed in the manuscript. This includes employment, consultancies, honoraria, stock ownership or options, expert testimony, grants or patents received or pending, or royalties.

No writing assistance was utilized in the production of this manuscript.

2 14th Annual Land O'Lakes Bioanalytical Conference.

http://ce.pharmacy.wisc.edu/ courseinfo/2013JulyLOL 